\title{
Analytic Solutions of an Iterative Functional Differential Equations Near Regular Points
}

\author{
Lingxia Liu \\ Department of Mathematics, Weifang University, Weifang, Shandong 261061, China \\ Correspondence should be addressed to Lingxia Liu; llxmath@126.com
}

Received 13 August 2012; Accepted 5 March 2013

Academic Editor: Juan Torregrosa

Copyright (C) 2013 Lingxia Liu. This is an open access article distributed under the Creative Commons Attribution License, which permits unrestricted use, distribution, and reproduction in any medium, provided the original work is properly cited.

The existence of analytic solutions of an iterative functional differential equation is studied when the given functions are all analytic and when the given functions have regular points. By reducing the equation to another functional equation without iteration of the unknown function an existence theorem is established for analytic solutions of the original equation.

\section{Introduction}

Functional differential equations with state-dependent delay have attracted the attentions of many authors in the last few years (see [1-8]). In [6-8], analytic solutions of the statedependent functional differential equations

$$
\begin{gathered}
x^{\prime}(z)=x(a z+b x(z)), \\
\alpha z+\beta x^{\prime}(z)=x\left(a z+b x^{\prime}(z)\right), \\
f^{m}(x)=G\left(\sum_{k=0}^{m-1} a_{k} f^{k}(x)\right)+F(x), \quad m \geq 2, x \in \mathbb{C}
\end{gathered}
$$

are found. In this paper, we will be concerned with analytic solutions of the functional differential equation

$$
\alpha z+\beta x^{\prime}(z)=F\left(\sum_{l=0}^{m} c_{l} x^{l}(z)\right)+G(z), \quad z \in \mathbb{C},
$$

where $\alpha, \beta, c_{1}, c_{2}, \ldots, c_{m}$ are complex numbers, $\beta \neq 0$, $\sum_{l=0}^{m}\left|c_{l}\right|<1$, and $x^{l}(z)=x\left(x^{l-1}(z)\right)$ that denote the $n$th iterate of a map $x$. In general, $F, G$ are given complex-valued functions of a complex variable.

In this paper, analytic solutions of nonlinear iterative functional differential equations are investigated. Existence of locally analytic solutions and their construction is given in the case that all given functions exist regular points. As well as in previous work [6-8], we still reduce this problem to find analytic solutions of a differential-difference equation and a functional differential equation with proportional delay. The existence of analytic solutions for such equation is closely related to the position of an indeterminate constant $\mu$ depending on the eigenvalue of the linearization of $x$ at its fixed point 0 in the complex plane. For technical reasons, in $[6,7]$, only the situation of $\mu$ off the unit circle in $\mathbb{C}$ and the situation of $\mu$ on the circle with the Diophantine condition, " $|\mu|=1, \mu$ is not a root of unity, and $\log \left(1 /\left|\mu^{n}-1\right|\right) \leq T \log n$, $n=2,3, \ldots$ for some positive constant $T$ ", are discussed. The Diophantine condition requires $\mu$ to be far from all roots of unity that the fixed point 0 is irrationally neutral. In this paper, besides the situation that $\mu$ is the inside of the unit circle $S^{1}$, we break the restriction of the Diophantine condition and study the situations that the constant $\mu$ in (5) (or $\mu=b^{-\lambda}, b$ is a complex constant, and $\lambda$ is in (4)) is resonance and a root of unity in the complex plane $\mathbb{C}$ near resonance under the Brjuno condition.

\section{Discussion on Auxiliary Equations}

In this section we assume that both $F$ and $G$ are analytic functions in a neighborhood of the origin, that is, 0 is a regular point, and have power series expansions

$$
\begin{gathered}
F(z)=\sum_{n=0}^{\infty} a_{n} z^{n}, \quad G(z)=\sum_{n=0}^{\infty} d_{n} z^{n}, \\
a_{0} \neq 0, \quad d_{0} \neq 0, \quad z \in \mathbb{C} .
\end{gathered}
$$


If there exists a complex constant $\lambda$ and an invertible function $\psi(z)$ such that $\psi\left(\psi^{-1}(z)+\lambda\right)$ is well defined, then letting $x(z)=\psi\left(\psi^{-1}(z)+\lambda\right)$, we can formally transform (2) into the differential-difference equation

$$
\begin{aligned}
\alpha \psi(z) \psi^{\prime}(z)+\beta \psi^{\prime}(z+\lambda)= & F\left(\sum_{l=0}^{m} c_{l} \psi(z+l \lambda)\right) \psi^{\prime}(z) \\
& +G(\psi(z)) \psi^{\prime}(z) .
\end{aligned}
$$

The indeterminate constant $\lambda$ will be discussed in the following cases:

(I1) $\mathfrak{R} \lambda>0$;

(I2) $\mu=b^{-\lambda}=e^{2 \pi i \theta}$, and $\theta \in \mathbb{R} \backslash \mathbb{Q}$ is a Brjuno number $[9,10]$; that is, $B(\theta)=\sum_{k=0}^{\infty}\left(\log \left(q_{k+1}\right) / q_{k}\right)<\infty$, where $\left\{p_{k} / q_{k}\right\}$ denotes the sequence of partial fraction of the continued fraction expansion of $\theta$, said to satisfy the Brjuno condition;

(I3) $\mu=b^{-\lambda}=e^{2 \pi i q / p}$ for some integers $p \in \mathbb{N}$ with $p \geq 2$ and $q \in \mathbb{Z} \backslash\{0\}$, and $\alpha \neq e^{2 \pi i l / k}$ for all $1 \leq k \leq p-1$ and $l \in \mathbb{Z} \backslash\{0\}$.

Take notations $S_{p}:=\{z \in \mathbb{C}: \mathfrak{R} z>-\ln \rho / \ln |b|,-\infty<$ $\Im z<+\infty\}$

A change of variable further transforms (4) into the functional differential equation

$$
\begin{aligned}
\alpha \varphi(z) \varphi^{\prime}(z)+\beta \mu \varphi^{\prime}(\mu z)= & F\left(\sum_{l=0}^{m} c_{l} \varphi\left(\mu^{l} z\right)\right) \varphi^{\prime}(z) \\
& +G(\varphi(z)) \varphi^{\prime}(z),
\end{aligned}
$$

where $\mu$ is a complex constant. The solution of this equation has properties similar to those of (4). If (5) has an invertible solution $\varphi(z)$, which satisfies the initial value conditions

$$
\varphi(0)=0, \quad \varphi^{\prime}(0)=\tau \neq 0,
$$

then we can show that $x(z)=\varphi\left(\mu \varphi^{-1}(z)\right)$ is an analytic solution of (2).

Theorem 1. Suppose that (I1) holds, then (5) has an analytic solution of the form

$$
\psi(z)=\sum_{n=1}^{\infty} b_{n} b^{-n z},
$$

in the half plane $S_{\rho}$ for a certain constant $\rho>0$, which satisfies $\lim _{\mathfrak{R} z \rightarrow+\infty} \psi(z)=0$.

Proof. Since $F$ and $G$ are analytic in a neighborhood of the origin and have the power series expansion (3), there exists a positive $\varrho$ such that

$$
\left|a_{n}\right| \leq e^{n-1}, \quad\left|d_{n}\right| \leq e^{n-1}, \quad n=2,3, \ldots .
$$

Without loss of generality, we can assume that $\varrho=1$; that is, $\left|a_{n}\right| \leq 1,\left|d_{n}\right| \leq 1$ for $n=2,3, \ldots$. In fact, let $\widetilde{F}(z)=\varrho F\left(\varrho^{-1} z\right)$,
$\widetilde{G}(z)=\varrho G\left(\varrho^{-1} z\right)$, and put $y=\varrho z, \widetilde{\beta}=\varrho \beta, u=\varrho \lambda$, and $\widetilde{\psi}(z)=\varrho \psi\left(\varrho^{-1} z\right)$. Then (4) can be rewritten as

$$
\begin{aligned}
\alpha \widetilde{\psi}(y) \widetilde{\psi}^{\prime}(y)+\widetilde{\beta} \widetilde{\psi}^{\prime}(y+u)= & \widetilde{F}\left(\sum_{l=0}^{m} c_{l} \widetilde{\psi}(y+l u)\right) \widetilde{\psi}^{\prime}(y) \\
& +\widetilde{G}(\widetilde{\psi}(y)) \widetilde{\psi}^{\prime}(y),
\end{aligned}
$$

in the same form as (4) and $\left|a_{n} \varrho^{1-n}\right| \leq 1,\left|d_{n} \varrho^{1-n}\right| \leq 1$ for $n=2,3, \ldots$ by $(8)$.

Consider a solution $\psi(z)$ of (4) in the formal Dirichlet series (7); that is, $\psi(z)=\sum_{n=1}^{\infty} b_{n} b^{-n z}$, where $b$ is a complex constant and $|b|>1$. Substituting series (3) and (7) of $F, G$, and $\psi$ in (4) and comparing coefficients we obtain that

$$
\begin{gathered}
{\left[\beta b^{-\lambda}-\left(a_{0}+d_{0}\right)\right] \ln b b_{1}=0,} \\
{\left[\beta b^{-n \lambda}-\left(a_{0}+d_{0}\right)\right] n b_{n}} \\
=-\alpha \sum_{i=1}^{n-1} i b_{i} b_{n-i} \\
+\sum_{i=1}^{n-1} \sum_{\substack{l_{1}+l_{2}+\cdots+l_{t}=n-i \\
t=1,2, \ldots, n-i}} i\left[d_{t}+a_{t} \prod_{k=1}^{t}\left(\sum_{l=0}^{m} c_{l} b^{l_{k} l \lambda}\right)\right] b_{i} b_{l_{1}} b_{l_{2}} \cdots b_{l_{t}},
\end{gathered}
$$

$n \geq 2$.

If $b_{1}=\tau=0$, then (4) has a trivial solution $\psi(z)=0$. Assume that $b_{1}=\tau \neq 0$, because $|b|>1$; from (10) we have $a_{0}+d_{0}=$ $\beta b^{-\lambda}$. From (11) we obtain that

$$
\begin{aligned}
\beta b^{-\lambda} & \left(b^{-(n-1) \lambda}-1\right) n b_{n} \\
= & -\alpha \sum_{i=1}^{n-1} i b_{i} b_{n-i} \\
& +\sum_{i=1}^{n-1} \sum_{\substack{l_{1}+l_{2}+\cdots+l_{t}=n-i \\
t=1,2, \ldots, n-i}} i\left[d_{t}+a_{t} \prod_{k=1}^{t}\left(\sum_{l=0}^{m} c_{l} b^{-l_{k} l \lambda}\right)\right] b_{i} b_{l_{1}} b_{l_{2}} \cdots b_{l_{t}},
\end{aligned}
$$

The sequence $\left\{b_{n}\right\}_{n=2}^{\infty}$ is successively determined by (12) in a unique manner.

In what follows we need to prove that the series (7) is convergent in a right-half plane. Since $\Re \lambda>0$, so we have

$$
\begin{gathered}
\lim _{n \rightarrow \infty}\left|\frac{\alpha i}{n \beta b^{-\lambda}\left(b^{-(n-1) \lambda}-1\right)}\right| \\
\quad \leq \lim _{n \rightarrow \infty} \frac{|\alpha|}{\left|\beta b^{-\lambda}\left(b^{-(n-1) \lambda}-1\right)\right|} \\
\quad=\frac{|\alpha|}{|\beta|\left|b^{-\lambda}\right|}, \quad \Re \lambda>0,
\end{gathered}
$$




$$
\begin{gathered}
\lim _{n \rightarrow \infty}\left|\frac{i\left[d_{t}+a_{t} \prod_{k=1}^{t}\left(\sum_{l=0}^{m} c_{l} b^{-l_{k} l \lambda}\right)\right]}{n \beta b^{-\lambda}\left(b^{-(n-1) \lambda}-1\right)}\right| \\
\leq \lim _{n \rightarrow \infty} \frac{\left|d_{t}\right|+\left|a_{t}\right| \prod_{k=1}^{t}\left(\sum_{l=0}^{m}\left|c_{l}\right|\right)}{\left|\beta b^{-\lambda}\left(b^{-(n-1) \lambda}-1\right)\right|} \\
\leq \frac{2}{|\beta|\left|b^{-\lambda}\right|}, \quad \Re \lambda>0 .
\end{gathered}
$$

This implies that there exists a constant $M>0$ such that

$$
\begin{gathered}
\lim _{n \rightarrow \infty}\left|\frac{\alpha i}{n \beta b^{-\lambda}\left(b^{-(n-1) \lambda}-1\right)}\right| \leq M, \\
\lim _{n \rightarrow \infty}\left|\frac{i\left[d_{t}+a_{t} \prod_{k=1}^{t}\left(\sum_{l=0}^{m} c_{l} b^{l_{k} l \lambda}\right)\right]}{n \beta b^{-\lambda}\left(b^{-(n-1) \lambda}-1\right)}\right| \leq M, \\
\forall n \geq 2, \quad \Re \lambda>0 .
\end{gathered}
$$

Therefore, from (12) we obtain

$$
\left|b_{n}\right| \leq M\left(\sum_{i=1}^{n-1}\left|b_{i}\right|\left|b_{n-i}\right|+\sum_{i=1}^{n-1} \sum_{\substack{l_{1}+l_{2}+\cdots++l_{t}=n-i \\ t=1,2, \ldots, n-i}}\left|b_{i}\right|\left|b_{l_{1}}\right|\left|b_{l_{2}}\right| \cdots\left|b_{l_{t}}\right|\right),
$$$$
n \geq 2 \text {. }
$$

In order to construct a majorant series of (7), we consider the implicit functional equation

$$
H(z)=|\tau| z+M\left[H^{2}(z)+\frac{H^{2}(z)}{1-H(z)}\right] .
$$

Define the function

$$
\omega(z, H):=\omega(z, H, \tau, M)=H-|\tau| z-M\left(H^{2}+\frac{H^{2}}{1-H}\right)
$$

for $(z, H)$ in a neighborhood of the origin. Then $\omega(0,0)=0$, $\omega_{H}^{\prime}(0,0)=1 \neq 0$. Thus, there exists a unique function $H(z)$ analytic in a neighborhood of zero; that is, there is a constant $\delta_{1}>0$, as $|z|<\delta_{1}$, the function $H(z)$ is analytic, such that $H(0)=0, H^{\prime}(0)=-\omega_{z}^{\prime}(0,0) / \omega_{H}^{\prime}(0,0)=|\tau|$ and $\omega(z, H(z))=$ 0 . Since $H(0)=0$, there is a constant $\delta_{2}>0$, such that $|H(z)|<1$ for $|z|<\delta_{2}$. Therefore, as $|z|<\delta:=\min \left\{\delta_{1}, \delta_{2}\right\}$, the function $H(z)$ satisfies the equation

$$
\omega(z, H(z))=H(z)-|\tau| z-M\left[H^{2}(z)+\frac{H^{2}(z)}{1-H(z)}\right]=0 .
$$

Choosing $B_{1}=|\tau|$ and putting

$$
H(z)=\sum_{n=1}^{\infty} B_{n} b^{-n z}
$$

in (18), we can determine all coefficients recursively by $B_{1}=$ $|\tau|$ and

$$
B_{n}=M\left(\sum_{i=1}^{n-1} B_{i} B_{n-i}+\sum_{i=1}^{n-1} \sum_{\substack{l_{1}+l_{2}+\cdots+l_{l}=n-i \\ t=1,2, \ldots, n-i}} B_{i} B_{l_{1}} B_{l_{2}} \cdots B_{l_{t}}\right),
$$

Moreover, it is easy to see from (15)

$$
\left|b_{n}\right| \leq B_{n}, \quad n=1,2, \ldots
$$

It follows that the power series

$$
\phi(z)=\sum_{n=1}^{\infty} b_{n} z^{n}
$$

is also convergent as $|z|<\delta$. So there exists $\rho \leq \delta$ such that Dirichlet series (7) is convergent in $S_{\rho}$.

Furthermore, one has

$$
\begin{aligned}
\lim _{\mathfrak{R} z \rightarrow+\infty} b^{-z} & =\lim _{\mathfrak{R} z \rightarrow+\infty} b^{-\mathfrak{R} z}[(\cos (\Im z \ln b))-i \sin (\Im z \ln b)] \\
& =0 .
\end{aligned}
$$

Thus $\lim _{\mathfrak{R} z \rightarrow+\infty} \psi(z)=\lim _{\mathfrak{R} z \rightarrow+\infty} \sum_{n=1}^{\infty} b_{n} b^{-n z}=0$. The proof is complete.

We observe that $\mu=b^{-\lambda}$ is inside the unit circle of (I1) but on the unit circle in the rest cases. Next we devote attention to the existence of analytic solutions of (4) under the Brjuno condition. To do this, we first recall briefly the definition of Brjuno numbers and some basic facts. As stated in [11], for a real number $\theta$, we let $[\theta]$ denote its integer part, and $\{\theta\}=$ $\theta-[\theta]$ its fractional part. Then every irrational number $\theta$ has a unique expression of the Gauss' continued fraction

$$
\theta=a_{0}+\theta_{0}=a_{0}+\frac{1}{a_{1}+\theta_{1}}=\cdots,
$$

denoted simply by $\theta=\left[a_{0}, a_{1}, \ldots, a_{n}, \ldots\right]$, where $a_{j}$ 's and $\theta_{j}$ 's are calculated by the algorithm: (a) $a_{0}=[\theta], \theta_{0}=\{\theta\}$ and (b) $a_{n}=\left[1 / \theta_{n-1}\right], \theta_{n}=\left\{1 / \theta_{n-1}\right\}$ for all $n \geq 1$. Define the sequences $\left(p_{n}\right)_{n \in \mathbb{N}}$ and $\left(q_{n}\right)_{n \in \mathbb{N}}$ as follows:

$$
\begin{array}{ll}
q_{-2}=1, & q_{-1}=0, \quad q_{n}=a_{n} q_{n-1}+q_{n-2}, \\
p_{-2}=0, & p_{-1}=1, \quad p_{n}=a_{n} p_{n-1}+p_{n-2} .
\end{array}
$$

It is easy to show that $p_{n} / q_{n}=\left[a_{0}, a_{1}, \ldots, a_{n}\right]$. Thus, for every $\theta \in \mathbb{R} \backslash \mathbb{Q}$ we associate, using its convergence, an arithmetical function $B(\theta)=\sum_{n \geq 0}\left(\log \left(q_{n+1}\right) / q_{n}\right)$. We say that $\theta$ is a Brjuno number or that it satisfies Brjuno condition if $B(\theta)<+\infty$. The Brjuno condition is weaker than the Diophantine condition. For example, if $a_{n+1} \leq c e^{a_{n}}$ for all $n \geq 0$, where $c>0$ is 
a constant, then $\theta=\left[a_{0}, a_{1}, \ldots, a_{n}, \ldots\right]$ is a Brjuno number but is not a Diophantine number. So, the case (I2) contains both Diophantine condition and a part of $\mu=b^{-\lambda}$ "near" resonance. let

$$
\begin{gathered}
A_{k}=\left\{n \geq 0 \mid\|n \theta\| \leq \frac{1}{8 q_{k}}\right\}, \quad E_{k}=\max \left(q_{k}, \frac{q_{k+1}}{4}\right), \\
\eta_{k}=\frac{q_{k}}{E_{k}} .
\end{gathered}
$$

Let $A_{k}^{*}$ be the set of integers $j \geq 0$ such that either $j \in A_{k}$ or for some $j_{1}$ and $j_{2}$ in $A_{k}$, with $j_{2}-j_{1}<E_{k}$, one has $j_{1}<j<j_{2}$ and $q_{k}$ divides $j-j_{1}$. For any integer $n \geq 0$, define

$$
l_{k}(n)=\max \left(\left(1+\eta_{k}\right) \frac{n}{q_{k}}-2,\left(m_{n} \eta_{k}+n\right) \frac{1}{q_{k}}-1\right),
$$

where $m_{n}=\max \left\{j \mid 0 \leq j \leq n, j \in A_{k}^{*}\right\}$. We then define the function $h_{k}: \mathbb{N} \rightarrow \mathbb{R}_{+}$as follows:

$$
h_{k}(n)= \begin{cases}\frac{m_{n}+\eta_{k} n}{q_{k}}-1, & \text { if } m_{n}+q_{k} \in A_{k}^{*} \\ l_{k}(n), & \text { if } m_{n}+q_{k} \notin A_{k}^{*}\end{cases}
$$

Let $g_{k}(n):=\max \left(h_{k}(n),\left[n / q_{k}\right]\right)$, and define $k(n)$ by the condition $q_{k(n)} \leq n \leq q_{k(n)+1}$. Clearly, $k(n)$ is nondecreasing. Moreover, the function $g_{k}$ is nonnegative. Then we are able to state the following result.

Lemma 2 (Davie's lemma [12]). Let $K(n)=n \log 2+$ $\sum_{j=0}^{k(n)} g_{j}(n) \log \left(2 q_{j+1}\right)$. Then

(a) there is a universal constant $\gamma>0$ (independent of $n$ and $\theta$ ) such that

$$
K(n) \leq n\left(\sum_{j=0}^{k(n)} \frac{\log q_{j+1}}{q_{j}}+\gamma\right),
$$

(b) $K\left(n_{1}\right)+K\left(n_{2}\right) \leq K\left(n_{1}+n_{2}\right)$ for all $n_{1}$ and $n_{2}$, and

(c) $-\log \left|b^{-\lambda n}-1\right| \leq K(n)-K(n-1)$.

Now we state and prove the following theorem under Brjuno condition.

Theorem 3. Suppose that (I2) holds. Then (4) has an analytic solution $\psi$ of the form (7) in the half plane $S_{\rho}=\{z \in \mathbb{C}: \mathfrak{R} z>$ $-\ln \rho / \ln |b|,-\infty<\Im z<+\infty\}$ for a certain constant $\rho>0$, which satisfies $\lim _{\mathfrak{R} z \rightarrow+\infty} \psi(z)=0$.

Proof. As in the proof of Theorem 1, we find a solution in the form of the Dirichlet series (7). Using the same method as above mentioned, for chosen $b_{1}=\tau$ we can uniquely determine the sequence $\left\{b_{n}\right\}_{n=2}^{\infty}$ recursively by (12). In fact, in view of (I2) we see that $\mu=b^{-\lambda}$ satisfies the conditions of Lemma 2, and from (12) we have

$$
\begin{aligned}
\left|b_{n}\right| \leq \frac{M_{1}}{\left|b^{-(n-1) \lambda}-1\right|} & \left(\sum_{i=1}^{n-1}\left|b_{i}\right|\left|b_{n-i}\right|\right. \\
& \left.+\sum_{i=1}^{n-1} \sum_{\substack{l_{1}+l_{2}+\cdots+l_{t}=n-i \\
t=1,2, \ldots, n-i}}\left|b_{i}\right|\left|b_{l_{1}}\right|\left|b_{l_{2}}\right| \cdots\left|b_{l_{t}}\right|\right),
\end{aligned}
$$

where $M_{1}=\max \{|\alpha| /|\beta|, 2 /|\beta|\}>0$.

To construct a governing series of (7), we consider the implicit functional equation

$$
\omega\left(z, U, \tau, M_{1}\right)=0
$$

where $\omega$ is defined in (17). Similarly to the proof of Theorem 1 , using the implicit function theorem we can prove that (31) has a unique analytic solution $U\left(z, \tau, M_{1}\right)$ in a neighborhood of the origin; that is, there is a constant $\delta_{3}>0$, as $|z|<\delta_{3}$, the function $U\left(z, \tau, M_{1}\right)$ is analytic such that $U\left(0, \tau, M_{1}\right)=0$ and $U_{z}^{\prime}\left(0, \tau, M_{1}\right)=|\tau|$. Thus $U\left(z, \tau, M_{1}\right)$ in (31) can be expanded into a convergent series

$$
U\left(z, \tau, M_{1}\right)=\sum_{n=1}^{\infty} C_{n} z^{n}
$$

in a neighborhood of the origin. Replacing (32) into (31) and comparing coefficients, we obtain that $C_{1}=|\tau|$ and

$$
C_{n}=M_{1}\left(\sum_{i=1}^{n-1} C_{i} C_{n-i}+\sum_{i=1}^{n-1} \sum_{\substack{l_{1}+l_{2}+\cdots+l_{t}=n-i \\ t=1,2, \ldots, n-i}} C_{i} C_{l_{1}} C_{l_{2}} \cdots C_{l_{t}}\right)
$$

Note that the series (32) converges in a neighborhood of the origin. So, there is a constant $T>0$ such that

$$
C_{n}<T^{n}, \quad n \geq 1
$$

Now, we can deduce, by induction, that $\left|b_{n}\right| \leq C_{n} e^{K(n-1)}$ for $n \geq 1$, where $K: \mathbb{N} \rightarrow \mathbb{R}$ is defined in Lemma 2. In 
fact $\left|b_{1}\right|=|\tau|=C_{1}$, for inductive proof, and we assume that $\left|b_{j}\right| \leq C_{j} e^{K(j-1)}, j \leq n$. From (30) and Lemma 2 we obtain

$$
\begin{aligned}
& \left|b_{n+1}\right| \\
& \leq \frac{M_{1}}{\left|b^{-n \lambda}-1\right|} \\
& \times\left(\sum_{i=1}^{n}\left|b_{i}\right|\left|b_{n-i+1}\right|\right. \\
& \left.+\sum_{i=1}^{n} \sum_{\substack{l_{1}+l_{2}+\cdots+l_{t}=n-i+1 \\
t=1,2, \ldots, n-i+1}}\left|b_{i}\right|\left|b_{l_{1}}\right|\left|b_{l_{2}}\right| \cdots\left|b_{l_{t}}\right|\right) \\
& \leq \frac{M_{1}}{\left|b^{-n \lambda}-1\right|}\left[\sum_{i=1}^{n} C_{i} C_{n-i+1} e^{K(i-1)+K(n-i)}\right. \\
& +\sum_{i=1}^{n} \sum_{\substack{l_{1}+l_{2}+\cdots+l_{t}=n-i+1 \\
t=1,2, \ldots, n-i+1}} C_{i} C_{l_{1}} C_{l_{2}} \cdots C_{l_{t}} \\
& \left.\times e^{K(i-1)+K\left(l_{1}-1\right)+\cdots+K\left(l_{t}-1\right)}\right] .
\end{aligned}
$$

Note that

$$
\begin{aligned}
& K(i-1)+K(n-i) \\
& \quad \leq K(n-1) \leq K(n)+\log \left|b^{-\lambda n}-1\right|, \\
& K\left(l_{1}-1\right)+K\left(l_{2}-1\right)+\cdots+K\left(l_{t}-1\right) \\
& \quad \leq K(n-i+1-t) \leq K(n-i), \\
& K(i-1)+K(n-i) \\
& \quad \leq K(n-1) \leq K(n)+\log \left|b^{-\lambda n}-1\right|,
\end{aligned}
$$

then

$$
\begin{aligned}
& \left|b_{n+1}\right| \\
& \leq \frac{e^{K(n-1)} M_{1}}{\left|b^{-n \lambda}-1\right|} \\
& \times\left(\sum_{i=1}^{n} C_{i} C_{n-i+1}+\sum_{i=1}^{n} \sum_{\substack{l_{1}+l_{2}+\cdots+l_{t}=n-i+1 \\
t=1,2, \ldots, n-i+1}} C_{i} C_{l_{1}} C_{l_{2}} \cdots C_{l_{t}}\right) \\
& \leq C_{n+1} e^{K(n)}
\end{aligned}
$$

as desired. Moreover, from Lemma 2, we know that $K(n) \leq$ $n(B(\theta)+\gamma)$ for some universal constant $\gamma>0$. Then from (34) we have $\left|b_{n}\right| \leq C_{n} e^{K(n-1)} \leq T^{n} e^{(n-1)(B(\theta)+\gamma)}$; that is, $\lim _{n \rightarrow \infty} \sup \left(\left|b_{n}\right|^{1 / n}\right) \leq \lim _{n \rightarrow \infty} \sup \left(T e^{((n-1) / n)(B(\theta)+\gamma)}\right)=$ $T e^{B(\theta)+\gamma}$, which shows that the series (7) converges for $|z|<$ $\rho=\min \left\{\delta_{3},\left(T e^{B(\theta)+\gamma}\right)^{-1}\right\}$. So does series (22) in $S_{\rho}$. Similarly $\lim _{\mathfrak{R} z \rightarrow+\infty} \psi(z)=0$, as proved in Theorem 1 .

The next theorem is devoted to the case of (I3), where $b^{-\lambda}$ is not only on the unit circle in $\mathbb{C}$ but also a root of unity. In this case the Diophantine condition and Brjuno condition are not satisfied. The idea of our proof is acquired from [13]. Let $\left\{A_{n}\right\}_{n=1}^{\infty}$ be a sequence defined by $A_{1}=\tau$ and

$A_{n}=\xi M_{1}\left(\sum_{i=1}^{n-1} A_{i} A_{n-i}+\sum_{i=1}^{n-1} \sum_{\substack{l_{1}+l_{2}+\cdots+l_{t}=n-i \\ t=1,2, \ldots, n-i}} A_{i} A_{l_{1}} A_{l_{2}} \cdots A_{l_{t}}\right)$,

$n \geq 2$,

where $\xi=\max \left\{1,\left|\alpha^{i}-1\right|^{-1}, i=1,2 \ldots, p-1\right\}$ and $M_{1}$ is defined in Theorem 3.

Theorem 4. Suppose that (I3) holds and $p$ is given as above mentioned. Let $\left\{b_{n}\right\}_{n=1}^{\infty}$ be determined recursively by $b_{1}=\tau$ and

$$
\beta b^{-\lambda}\left(b^{-(n-1) \lambda}-1\right) n b_{n}=\Omega(n, \lambda), \quad n=2,3, \ldots,
$$

where

$\Omega(n, \lambda)$

$$
\begin{aligned}
= & -\alpha \sum_{i=1}^{n-1} i b_{i} b_{n-i} \\
& +\sum_{i=1}^{n-1} \sum_{\substack{l_{1}+l_{2}+\cdots+l_{t}=n-i \\
t=1,2, \ldots, n-i}} i\left[d_{t}+a_{t} \prod_{k=1}^{t}\left(\sum_{l=0}^{m} c_{l} b^{-l_{k} l \lambda}\right)\right] b_{i} b_{l_{1}} b_{l_{2}} \cdots b_{l_{t}} .
\end{aligned}
$$

If $\Omega(\nu p+1, \lambda)=0$ for all $\nu=1,2, \ldots$, then (4) has an analytic solution $\psi(z)=\phi\left(b^{-z}\right)$ in the half plane $S_{\rho}:=\{z \in \mathbb{C}: \mathfrak{R} z>$ $-\ln \rho / \ln |b|,-\infty<\Im z<+\infty\}$ for a certain $\rho>0$, where $\phi$ is an analytic function of the form (22) in $U_{\rho}(0)=\{z|| z \mid<\rho\}$ such that $\phi(0)=0$, and $\phi^{(\nu p+1)}(0)=(\nu p+1) ! \tau_{\nu p+1}$, for all $\nu=$ $0,1,2, \ldots$, where $\tau_{\nu p+1}^{\prime} s$ are arbitrary constants satisfying the inequality $\left|\tau_{\nu p+1}\right| \leq A_{\nu p+1}$ and the sequence $\left\{A_{n}\right\}_{n=1}^{\infty}$ is defined in (38). The other derivatives at 0 satisfy that $\phi^{(i)}(0)=i ! b_{i}$ for $i \neq \nu p+1$. Otherwise, if $\Omega(\nu p+1, \lambda) \neq 0$ for some $\nu=1,2, \ldots$, then (4) has no analytic solutions in the half plane $S_{\rho}:=\{z \in$ $\mathbb{C}: \mathfrak{R} z>-\ln \rho / \ln |b|,-\infty<\mathfrak{I} z<+\infty\}$ for any $\rho>0$.

Proof. Analogously to the proof of Theorem 1, we seek for a solution of (4) in Dirichlet (7). Without loss of generality, as in the proof of Theorem 1 we still assume that $\rho=1$ in (8). Taking (3) and (7) in (4) and defining $b_{1}=\tau \neq 0$, we obtain (12) or (39). If $\Omega(\nu p+1, \lambda)=0$ for all natural numbers $\nu$, then for each $\nu, b^{-v p \lambda}-1=0$, the corresponding $b_{v p+1}$ has infinitely many choices in $\mathbb{C}$; that is, the formal series solution 
(7) or (22) defines a family of solutions with infinitely many parameters. Choose $b_{v p+1}=\tau_{v p+1}$ arbitrarily such that

$$
\left|\tau_{v p+1}\right| \leq A_{v p+1}, \quad v=0,1,2, \ldots,
$$

where $A_{\nu p+1}$ is defined by (38). In what follows, we prove that the formal series solution (7) converges in a neighborhood of the origin. Observe that $\left|b^{-n \lambda}-1\right|^{-1} \leq \xi$ for $n \neq v p$. It follows from (12) or (39) that

$$
\begin{aligned}
\left|b_{n}\right| \leq \xi M_{1}( & \sum_{i=1}^{n-1}\left|b_{i}\right|\left|b_{n-i}\right| \\
& \left.+\sum_{i=1}^{n-1} \sum_{\substack{l_{1}+l_{2}+\cdots+l_{t}=n-i \\
t=1,2, \ldots, n-i}}\left|b_{i}\right|\left|b_{l_{1}}\right|\left|b_{l_{2}}\right| \cdots\left|b_{l_{t}}\right|\right)
\end{aligned}
$$

for all $n \neq v p+1, v=0,1,2, \ldots$, where $M_{1}$ is defined in Theorem 3. Let

$$
V\left(z, \tau, \xi M_{1}\right)=\sum_{n=1}^{\infty} A_{n} z^{n}, \quad A_{1}=|\tau| .
$$

It is easy to check that there exists a constant $\rho>0$, for $|z|<\rho$, and (43) satisfies the implicit functional equation

$$
\omega\left(z, V, \tau, \xi M_{1}\right)=0,
$$

where $\omega$ is defined in (17). Moreover, similarly to the proof of Theorem 1, we can prove that (44) has a unique analytic solution $V\left(Z, \tau, \xi M_{1}\right)$ in a neighborhood of the origin such that $V\left(0, \tau, \xi M_{1}\right)=0$ and $V_{z}^{\prime}\left(0, \tau, \xi M_{1}\right)=|\tau| \neq 0$. Thus (43) converges in a neighborhood of the origin. Moreover, it is easy to show that, by induction,

$$
\left|b_{n}\right| \leq A_{n}, \quad n=1,2, \ldots
$$

By inequality (45) we see that the series (22) converges in $U_{\rho}(0)=\{z|| z \mid<\rho\}$. Thus series (7) converges in $S_{\rho}$. This completes the proof.

The following theorem shows that each analytic solution of (4) leads to an analytic solution of (5). We shall discuss (5) in the following cases:

(H1) $0<|\mu| \neq 1$;

(H2) $\mu=e^{2 \pi i \theta}$, where $\theta \in \mathbb{R} \backslash \mathbb{Q}$ is a Brjuno number, $B(\theta)=$ $\sum_{k=0}^{\infty}\left(\log \left(q_{k+1}\right) / q_{k}\right)<\infty$, where $\left\{p_{k} / q_{k}\right\}$ denotes the sequence of partial fraction of the continued fraction expansion of $\theta$, said to satisfy the Brjuno condition;

(H3) $\mu=e^{2 \pi i q / p}$ for some integers $p \in \mathbb{N}$ with $p \geq 2$ and $q \in \mathbb{Z} \backslash\{0\}$, and $\mu \neq e^{2 \tau i l / k}$ for all $1 \leq k \leq p-1$ and $l \in \mathbb{Z} \backslash\{0\}$.

Theorem 5. Suppose that (H1) holds and that $a_{0} \neq 0, d_{0} \neq 0$. Then in a neighborhood of the origin (5) has an analytic solution $\varphi$ satisfying $\varphi(0)=0, \varphi^{\prime}(0)=\eta$.
Proof. Let

$$
\varphi(z)=\sum_{n=1}^{\infty} b_{n} z^{n}, \quad b_{1}=\eta
$$

be the formal series of the solution $\varphi$ for (5). We are going to determine $\left\{b_{n}\right\}_{n=1}^{\infty}$. Substituting (3) and (46) into (5) and comparing coefficients, we obtain

$$
\begin{gathered}
{\left[\beta \mu-\left(a_{0}+d_{0}\right)\right] b_{1}=0} \\
(n+1)\left[\beta \mu^{n+1}-\left(a_{0}+d_{0}\right)\right] b_{n+1} \\
=-\alpha \sum_{i=0}^{n-1}(i+1) b_{i+1} b_{n-i} \\
+\sum_{i=0}^{n-1} \sum_{\substack{l_{1}+l_{2}+\cdots+l_{t}=n-i \\
t=1,2, \ldots, n-i}}(i+1)\left[d_{t}+a_{t} \prod_{k=1}^{t}\left(\sum_{l=0}^{m} c_{l} \mu^{l l_{k}}\right)\right] \\
\times b_{i+1} b_{l_{1}} b_{l_{2}} \cdots b_{l_{t}}, \quad n \geq 1 .
\end{gathered}
$$

If $b_{1}=\eta=0$, then (5) has a trivial solution $\varphi(z)=0$. Assume that $b_{1}=\eta \neq 0$; from (47) we can choose $\beta \mu=a_{0}+d_{0}$, then (48) can be changed into

$$
\begin{aligned}
(n+1) & \beta \mu\left(\mu^{n}-1\right) b_{n+1} \\
=-\alpha \sum_{i=0}^{n-1}(i+1) b_{i+1} b_{n-i} & \\
+ & \sum_{\substack{i=0 \\
l_{1}+l_{2}+\ldots+l_{t}=n-i \\
t=1,2, \ldots, n-i}}^{n-1}(i+1)\left[d_{t}+a_{t} \prod_{k=1}^{t}\left(\sum_{l=0}^{m} \mathcal{c}_{l} \mu^{l l_{k}}\right)\right] \\
& \quad \times b_{i+1} b_{l_{1}} b_{l_{2}} \cdots b_{l_{t}}, \quad n \geq 1 .
\end{aligned}
$$

From (49) the sequence $\left\{b_{n}\right\}_{n=2}^{\infty}$ is determined uniquely in the recursive way.

Now we show the convergence of series (46) near zero. Since the power series in (3) are both convergent for $|z|<\sigma$, for any fixed $r \in(0, \sigma)$ there exists a constant $M_{2}>0$ such that

$$
\left|a_{n}\right| \leq \frac{M_{2}}{r^{n}}, \quad\left|d_{n}\right| \leq \frac{M_{2}}{r^{n}} .
$$

Note that since $1 \leq i \leq n-1$, then there exists some positive number $M_{3}$ as follows:

$$
\begin{gathered}
\left|\frac{(i+1) \prod_{k=1}^{t}\left(\sum_{l=0}^{m} c_{l} \mu^{l l_{k}}\right)}{(n+1) \beta \mu\left(\mu^{n}-1\right)}\right| \leq \frac{1}{|\beta||\mu|\left|\mu^{n}-1\right|} \leq M_{3}, \\
\left|\frac{i+1}{(n+1) \beta \mu\left(\mu^{n}-1\right)}\right| \leq \frac{1}{|\beta||\mu|\left|\mu^{n}-1\right|} \leq M_{3}, \\
\left|\frac{\alpha(i+1)}{(n+1) \beta \mu\left(\mu^{n}-1\right)}\right| \leq \frac{|\alpha|}{|\beta||\mu|\left|\mu^{n}-1\right|} \leq M_{3},
\end{gathered}
$$


then we have

$$
\begin{aligned}
& \left|b_{n+1}\right| \\
& \leq L\left(\sum_{i=0}^{n-1}\left|b_{i+1}\right|\left|b_{n-i}\right|\right. \\
& \left.+\sum_{i=0}^{n-1} \sum_{\substack{l_{1}+l_{2}+\cdots+l_{t}=n-i \\
t=1,2, \ldots, n-i}} \frac{1}{r^{t}}\left|b_{i+1}\right|\left|b_{l_{1}}\right|\left|b_{l_{2}}\right| \cdots\left|b_{l_{t}}\right|\right), \\
& n \geq 1 \text {, }
\end{aligned}
$$

where $L:=\max \left\{2 M_{2} M_{3}, M_{3}\right\}>0$. Let

$$
\Theta(z, W)=W-|\eta| z-L\left(\frac{W^{2} / r}{1-(W / r)}+W^{2}\right)
$$

for $(z, W)$ from a neighborhood of the origin. Since $\Theta(0,0)=$ $0, \Theta_{W}^{\prime}(0,0)=1 \neq 0$, there exists a unique function $W(z)$, analytic in a neighborhood of the origin, such that $W(0)=0$, $W^{\prime}(0)=|\eta| \neq 0$, and $\Theta(z, W(z))=0$. Then define a sequence $\left\{D_{n}\right\}_{n=1}^{\infty}$ by $D_{1}=|\eta| \neq 0$ and

$$
\begin{aligned}
D_{n+1}=L( & \left(\sum_{i=0}^{n-1} D_{i+1} D_{n-i}\right. \\
& \left.\quad+\sum_{i=0}^{n-1} \sum_{\substack{l_{1}+l_{2}+\cdots+l_{t}=n-i \\
t=1,2, \ldots, n-i}} \frac{1}{r^{t}} D_{i+1} D_{l_{1}} D_{l_{2}} \cdots D_{l_{t}}\right),
\end{aligned}
$$

By (52) we see that

$$
\left|b_{n}\right| \leq D_{n}, \quad n \geq 1
$$

Let

$$
P(z)=\sum_{n=1}^{\infty} D_{n} z^{n}, \quad D_{1}=|\eta|
$$

with the recursive law of $\left\{D_{n}\right\}_{n=1}^{\infty}$. Then

$$
\begin{aligned}
P^{2} & (z) \\
& =\left(\sum_{n=0}^{\infty} D_{n+1} z^{n+1}\right)\left(\sum_{n=1}^{\infty} D_{n} z^{n}\right) \\
& =\sum_{n=1}^{\infty} \sum_{i=0}^{n-1} D_{i+1} D_{n-i} z^{n+1},
\end{aligned}
$$

$$
\begin{aligned}
& \frac{P^{2}(z) / r}{1-(P(z) / r)} \\
& =P(z) \frac{P(z) / r}{1-(P(z) / r)} \\
& =\left(\sum_{n=0}^{\infty} D_{n+1} z^{n+1}\right)\left(\sum_{n=1}^{\infty}\left(\frac{P(z)}{r}\right)^{n}\right) \\
& =\left(\sum_{n=0}^{\infty} D_{n+1} z^{n+1}\right)\left(\sum_{n=1}^{\infty} \frac{1}{r^{n}}\left(\sum_{n=1}^{\infty} D_{n} z^{n}\right)^{n}\right) \\
& =\left(\sum_{n=0}^{\infty} D_{n+1} z^{n+1}\right)\left(\sum_{\substack{n=1 \\
l_{1}+l_{2}+\cdots+l_{t}=n \\
t=1,2, \ldots, n}}^{\infty} \frac{1}{r^{t}} D_{l_{1}} D_{l_{2}} \cdots D_{l_{t}} z^{n}\right) \\
& =\sum_{n=1}^{\infty} \sum_{\substack{i=0 \\
l_{1}+l_{2}+\cdots+l_{t}=n-i \\
t=1,2, \ldots, n-i}}^{n-1} \frac{1}{r^{t}} D_{i+1} D_{l_{1}} D_{l_{2}} \cdots D_{l_{t}} z^{n+1} .
\end{aligned}
$$

Then we have

$$
\frac{P^{2}(z) / r}{1-(P(z) / r)}+p^{2}(z)=\frac{1}{L} \sum_{n=1}^{\infty} D_{n+1} z^{n+1}=\frac{1}{L}(P(z)-|\eta| z),
$$

that is

$$
P(z)-|\eta| z-L\left[\frac{P^{2}(z) / r}{1-(P(z) / r)}+p^{2}(z)\right]=0 .
$$

This shows that $P(0)=0, P^{\prime}(0)=|\eta|$, and $\Theta(z, P(z))=0$. So we have $P(z)=W(z)$. It follows that the power series (56) converges in a neighborhood of the origin. Therefore, from (55) we see that (46) converges in a neighborhood of the origin. The proof is complete.

In the case (H2) we obtain similarly an analogue to Theorem 3.

Theorem 6. Suppose that (H2) holds and that $a_{0} \neq 0, d_{0} \neq 0$. Then in a neighborhood of the origin (5) has an analytic solution $\varphi(z)$ satisfying $\varphi(0)=0, \varphi^{\prime}(0)=\eta \neq 0$.

In the case (H3) we also obtain similarly an analogue to Theorem 4.

Theorem 7. Suppose that (H3) holds, $a_{0} \neq 0, d_{0} \neq 0$, and $p$ is given as above mentioned. Let $\left\{b_{n}\right\}_{n=1}^{\infty}$ be determined recursively by $b_{1}=\eta$ and

$$
(n+1) \beta \mu\left(\mu^{n}-1\right) b_{n+1}=\Xi(n+1, \mu),
$$


where

$$
\begin{aligned}
& \Xi(n+1, \mu) \\
& =-\alpha \sum_{i=0}^{n-1}(i+1) b_{i+1} b_{n-i} \\
& +\sum_{\substack{i=0 \\
l_{1}+l_{2}+\cdots+l_{t}=n-i \\
t=1,2, \ldots, n-i}}^{n-1}(i+1)\left[d_{t}+a_{t} \prod_{k=1}^{t}\left(\sum_{l=0}^{m} c_{l} \mu^{l l_{k}}\right)\right] \\
& \quad \times b_{i+1} b_{l_{1}} b_{l_{2}} \cdots b_{l_{t}}, \quad n \geq 1 .
\end{aligned}
$$

If $\Xi(l p+1, \mu)=0$ for all $l=1,2, \ldots$, then (5) has an analytic solution in a neighborhood of the origin such that $\varphi(0)=0$, $\varphi^{\prime}(0)=\eta \neq 0$, and $\varphi^{(l p+1)}(0)=(l p+1) ! \eta_{l p+1}$, where $\eta_{l p+1}^{\prime}$ s are arbitrary constants satisfying the inequality $\left|\eta_{l p+1}\right| \leq d_{l p+1}, l=$ $1,2, \ldots$ and the sequence $\left\{d_{n}\right\}_{n=1}^{\infty}$ is defined as follows: $d_{1}=|\eta|$ and

$d_{n+1}$

$$
=\Gamma L_{1}\left(\sum_{i=0}^{n-1} d_{i+1} d_{n-i}+\sum_{i=0}^{n-1} \sum_{\substack{l_{1}+l_{2}+\cdots+l_{t}=n-i \\ t=1,2, \ldots, n-i}} \frac{1}{r^{t}} d_{i+1} d_{l_{1}} d_{l_{2}} \cdots d_{l_{t}}\right),
$$

where $\Gamma=\max \left\{1,\left|\mu^{i}-1\right|^{-1}, i=1,2 \ldots, p-1\right\}$, and $L_{1}=$ $\max \{2 /|\beta|,|\alpha| /|\beta|\}$. Otherwise, if $\Xi(l p+1, \mu) \neq 0$ for some $l=$ $1,2, \ldots$, then (5) has no analytic solutions in any neighborhood of the origin.

\section{Analytic Solutions of the Original (2)}

Having known analytic solutions of the auxiliary equation (4) and (5), we can give results to the original (2).

Theorem 8. Suppose that the conditions of Theorems 1, 3, or 4 are satisfied. Then (2) has an analytic solution $x(z)=$ $\psi\left(\psi^{-1}(z)+\lambda\right)$ in a neighborhood of the origin, where $\psi(z)$ is an analytic solution of $(4)$ in the half plane $S_{\rho}$.

Proof. In view of Theorems 1, 3, or 4, we may find a sequence $\left\{b_{n}\right\}_{n=1}^{\infty}$ such that the function $\psi(z)$ of the form (7) is an analytic solution of (4) in the half plane $S_{\rho}$. As in Theorem 1, $\psi(z)=\phi\left(b^{-z}\right)$. Since $\phi^{\prime}(0) \neq 0$, the function $\phi^{-1}$ is analytic in a neighborhood of the point $\phi(0)=0$. Thus $\psi^{-1}(z)=$ $-\ln \phi^{-1}(z) / \ln b$ is analytic in a neighborhood of the origin. Define $x(z)=\psi\left(\psi^{-1}(z)+\lambda\right)$ which is analytic clearly. Note that

$$
x^{\prime}(z)=\frac{\psi^{\prime}\left(\psi^{-1}(z)+\lambda\right)}{\psi^{\prime}\left(\psi^{-1}(z)\right)}, \quad x^{k}(z)=\psi\left(\psi^{-1}(z)+k \lambda\right),
$$

then from (4)

$$
\begin{aligned}
\alpha z+\beta x^{\prime}(z) & =\alpha z+\frac{\beta \psi^{\prime}\left(\psi^{-1}(z)+\lambda\right)}{\psi^{\prime}\left(\psi^{-1}(z)\right)} \\
& =F\left(\sum_{l=0}^{m} c_{l} \psi\left(\psi^{-1}(z)+l \lambda\right)\right)+G\left(\psi\left(\psi^{-1}(z)\right)\right) \\
& =F\left(\sum_{l=0}^{m} c_{l} x^{l}(z)\right)+G(z) .
\end{aligned}
$$

This shows that $x(z)=\psi\left(\psi^{-1}(z)+\lambda\right)$ satisfies (2). The proof is complete.

Under the hypothesis of Theorem 1 the origin 0 is a hyperbolic fixed point of $x$, but under hypotheses of Theorems 3 and 4 it is not. Actually, when the constant $\mu=b^{-\lambda}$ satisfies the Brjuno condition, the norm of the eigenvalue of the linearized of $x$ at 0 equals 1 , but the eigenvalue is not a root of unity. Under (I3), the fixed point 0 of $x$ is a resonance.

When $0<|b|<1$, the same method is applicable and a similarly result can be obtained; that is, there exists a constant $\rho>0$ such that (4) has an analytic solution in the left-half plane $\{z \in \mathbb{C}|\mathfrak{R} z<-\ln \rho / \ln | b \mid,-\infty<\Im z<+\infty\}$.

Theorem 9. Under one of the conditions in Theorems 5, 6, or 7 , (2) has an analytic solution of the form $x(z)=\varphi\left(\mu \varphi^{-1}(z)\right)$ in a neighborhood of the origin, where $\varphi(z)$ is an analytic solution of (5) in a neighborhood of the origin.

Proof. In Theorems 5-7 we have found a solution $\varphi(z)$ of (5) in the form (46), which is analytic near 0 . Since $\varphi(0)=0$, $\varphi^{\prime}(0)=\eta \neq 0$, the function $\varphi^{-1}$ is also analytic near 0 . Thus $x(z)=\varphi\left(\mu \varphi^{-1}(z)\right)$ is analytic. Moreover,

$$
x^{\prime}(z)=\frac{\mu \varphi^{\prime}\left(\mu \varphi^{-1}(z)\right)}{\varphi^{\prime}\left(\varphi^{-1}(z)\right)}, \quad x^{l}(z)=\varphi\left(\mu^{l} \varphi^{-1}(z)\right),
$$

so from (5) we have

$$
\begin{aligned}
\alpha z+\beta x^{\prime}(z) & =\alpha z+\frac{\beta \mu \varphi^{\prime}\left(\mu \varphi^{-1}(z)\right)}{\varphi^{\prime}\left(\varphi^{-1}(z)\right)} \\
& =F\left(\sum_{l=0}^{m} c_{l} \varphi\left(\mu^{l} \varphi^{-1}(z)\right)\right)+G\left(\varphi\left(\varphi^{-1}(z)\right)\right) \\
& =F\left(\sum_{l=0}^{m} c_{l} x^{l}(z)\right)+G(z) .
\end{aligned}
$$

Therefore, $x(z)=\varphi\left(\mu \varphi^{-1}(z)\right)$ satisfies (2). The proof is complete. 


\section{Example}

Example 1. Consider the equation

$$
z+x^{\prime}(z)=4 e^{(1 / 2) x(x(z))}-e^{z}-\frac{17}{6} .
$$

It is in the form of (2), where $\alpha=\beta=1, c_{0}=c_{1}=0, c_{2}=1 / 2$, $m=2, F(z)=4 e^{z}-(13 / 3)=\sum_{n=1}^{\infty}\left(4 z^{n} / n !\right)-(1 / 3)$, and $G(z)=(3 / 2)-e^{z}=-\sum_{n=1}^{\infty}\left(z^{n} / n !\right)+(1 / 2)$. Clearly both $G$ and $F$ are analytic near $0, a_{0}=F(0)=-1 / 3, d_{0}=G(0)=1 / 2, a_{1}=$ $F^{\prime}(0)=4$, and $d_{1}=G^{\prime}(0)=-1$. For arbitrary $|b|>1$, let $\lambda=$ $\ln 6 / \ln |b|$, the $0<\left|b^{-\lambda}\right|<1$. By Theorem 1 , there is a constant $\rho>0$ such that the corresponding auxiliary equation

$$
\begin{aligned}
& \psi(z) \psi^{\prime}(z)+\psi^{\prime}(z+\lambda) \\
& =F\left(\frac{1}{2} \psi(z+2 \lambda)\right) \psi^{\prime}(z)+G(\psi(z)) \psi^{\prime}(z),
\end{aligned}
$$

and (67) itself have an analytic solution in the half plane $S_{\rho}=$ $\{z \mid \mathfrak{R} z>-(\ln \rho / \ln b),-\infty<\mathfrak{\Im} z<+\infty\}$. In the routine in the proofs of our theorems we can calculate the solutions

$$
x(z)=\frac{1}{6} z-\frac{35}{36} z^{2}-\frac{551}{1944} z^{3}+\cdots
$$

Example 2. Consider the equation

$$
z-x^{\prime}(z)=\frac{1}{2} x(z)-\frac{1}{4} x(x(z))+e^{z}-\frac{1}{4} .
$$

It is in the form of (2), where $\alpha=1, \beta=-1, c_{0}=0, c_{1}=-1 / 2$, $c_{2}=1 / 4, m=2, a_{0}=1 / 2, a_{1}=-1, a_{n}=0(n \geq 2)$, $d_{0}=1 / 4, d_{1}=1, F(z)=-z+(1 / 2)$, and $G(z)=e^{z}-(3 / 4)=$ $\sum_{n=1}^{\infty}\left(z^{n} / n !\right)+(1 / 4)$. Clearly, $\sum_{l=0}^{2}\left|c_{l}\right|<1$ and $\mu=(1 / \beta)\left(a_{0}+\right.$ $\left.d_{0}\right)=-3 / 4$ such that $0<|\mu|<1$. By Theorem 5 the corresponding auxiliary equation

$$
\begin{aligned}
\varphi(z) \varphi^{\prime}(z)-\mu \varphi^{\prime}(\mu z)= & \left(\frac{1}{2} \varphi(\mu z)-\frac{1}{4} \varphi\left(\mu^{2} z\right)\right) \varphi^{\prime}(z) \\
& +\left(e^{\varphi(z)}-\frac{1}{4}\right) \varphi^{\prime}(z)
\end{aligned}
$$

and (70) itself have an analytic solution each in a neighborhood of the origin. Proofs of our theorems provide a method to calculate the solution

$$
\begin{aligned}
x(z)= & \mu z+\frac{1}{8} \mu(\mu-2) z^{2} \\
& +\frac{1}{3 !}\left[\frac{1}{16} \mu(\mu-1)(\mu-2)(\mu+2)-1\right] z^{3}+\cdots .
\end{aligned}
$$

\section{Acknowledgments}

This work was supported by the Natural Science Foundation of Shandong Province (ZR2012AM017) and the Natural Science Foundation of Shandong Province (2011ZRA07006).

\section{References}

[1] K. Wang, "On the equation $x^{\prime}(t)=f(x(x(t)))$," Funkcialaj Ekvacioj, vol. 33, no. 3, pp. 405-425, 1990.

[2] S. Stanck, "On global properties of solutions of functional differential equation $x^{\prime}(t)=x(x(t))+x(t)$," Dynamic Systems and Applications, vol. 4, pp. 263-278, 1995.

[3] J.-G. Si, W.-R. Li, and S. S. Cheng, "Analytic solutions of an iterative functional-differential equation," Computers \& Mathematics with Applications, vol. 33, no. 6, pp. 47-51, 1997.

[4] J.-G. Si and S. S. Cheng, "Smooth solutions of a nonhomogeneous iterative functional-differential equation," Proceedings of the Royal Society of Edinburgh Section A, vol. 128, no. 4, pp. 821831, 1998.

[5] J.-G. Si and X.-P. Wang, "Smooth solutions of a nonhomogeneous iterative functional-differential equation with variable coefficients," Journal of Mathematical Analysis and Applications, vol. 226, no. 2, pp. 377-392, 1998.

[6] J.-G. Si and S. S. Cheng, "Analytic solutions of a functional-differential equation with state dependent argument," Taiwanese Journal of Mathematics, vol. 1, no. 4, pp. 471-480, 1997.

[7] J.-G. Si, X.-P. Wang, and S. S. Cheng, "Analytic solutions of a functional-differential equation with a state derivative dependent delay," Aequationes Mathematicae, vol. 57, no. 1, pp. 75-86, 1999.

[8] J. Si and W. Zhang, "Analytic solutions of a nonlinear iterative equation near neutral fixed points and poles," Journal of Mathematical Analysis and Applications, vol. 284, no. 1, pp. 373-388, 2003.

[9] A. D. Bjuno, "Analytic form of differential equations," Transactions of the Moscow Mathematical Society, vol. 25, pp. 131-288, 1971.

[10] S. Marmi, P. Moussa, and J.-C. Yoccoz, "The Brjuno functions and their regularity properties," Communications in Mathematical Physics, vol. 186, no. 2, pp. 265-293, 1997.

[11] T. Carletti and S. Marmi, "Linearization of analytic and nonanalytic germs of diffeomorphisms of $(\mathbb{C}, 0)$, , Bulletin de la Société Mathématique de France, vol. 128, no. 1, pp. 69-85, 2000.

[12] A. M. Davie, "The critical function for the semistandard map," Nonlinearity, vol. 7, no. 1, pp. 219-229, 1994.

[13] D. Bessis, S. Marmi, and G. Turchetti, "On the singularities of divergent majorant series arising from normal form theory," Rendiconti di Matematica e delle sue Applicazioni, vol. 9, no. 4, pp. 645-659, 1989. 


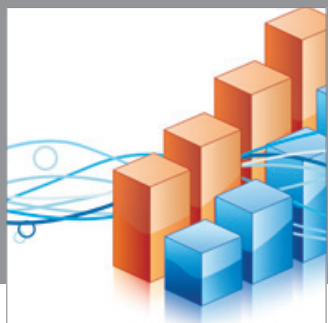

Advances in

Operations Research

mansans

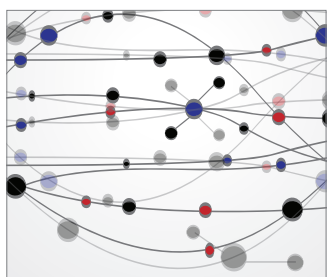

The Scientific World Journal
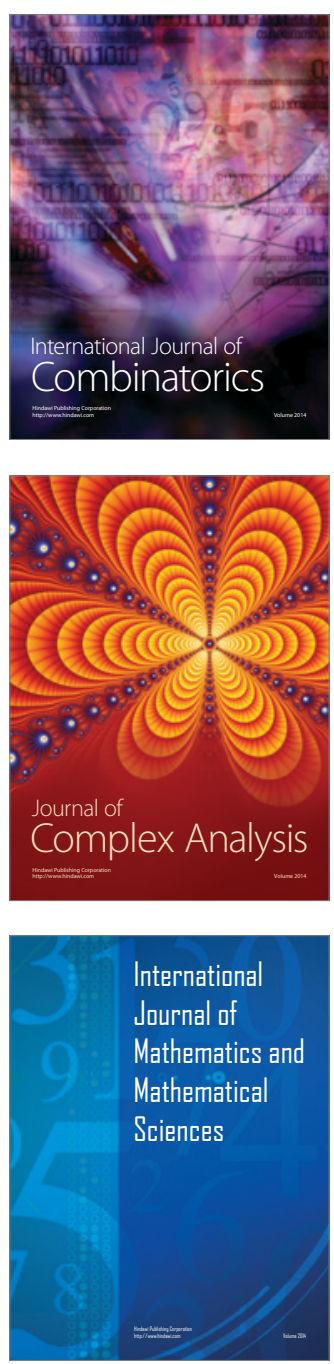
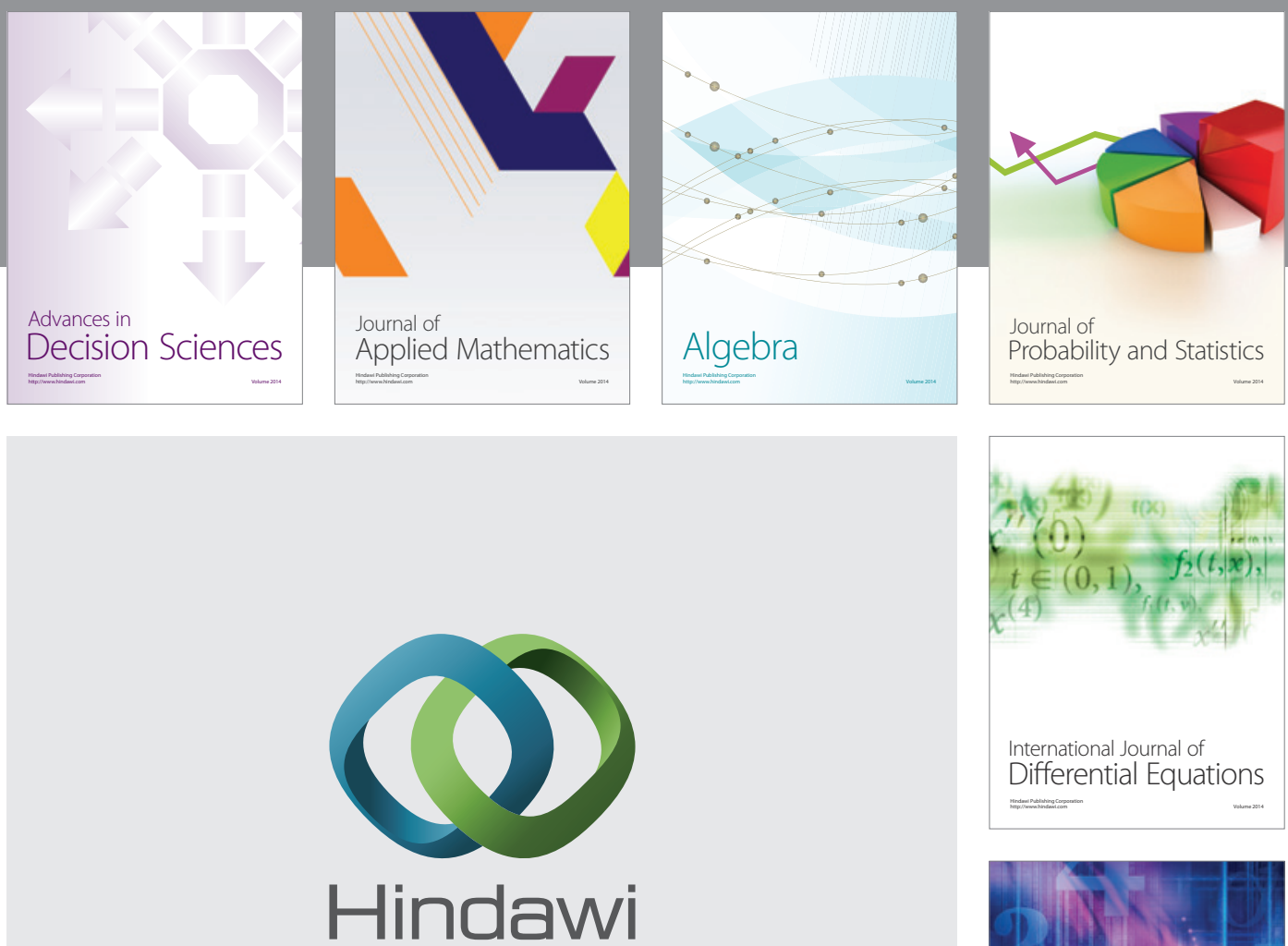

Submit your manuscripts at http://www.hindawi.com
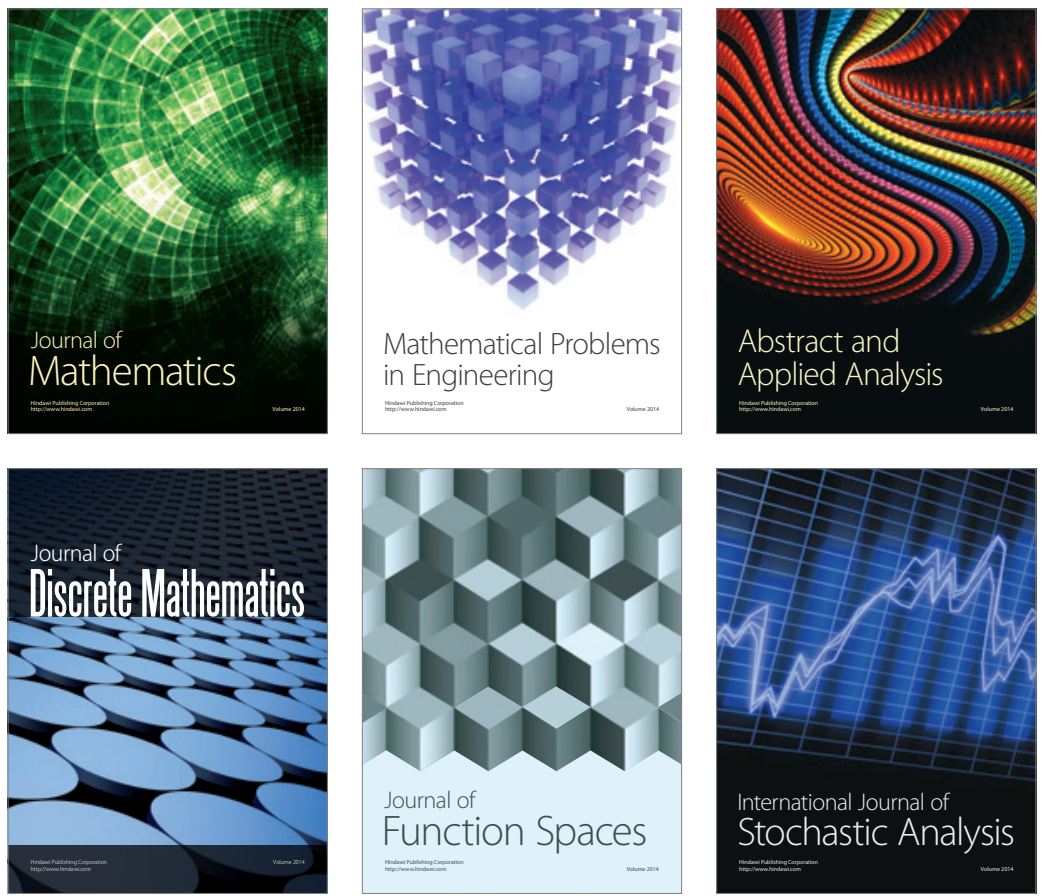

Journal of

Function Spaces

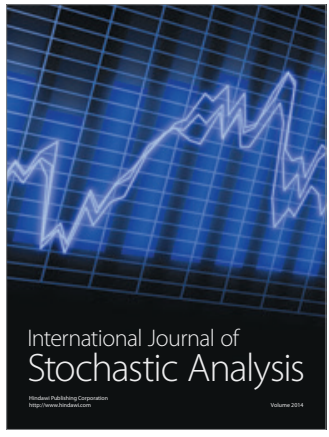

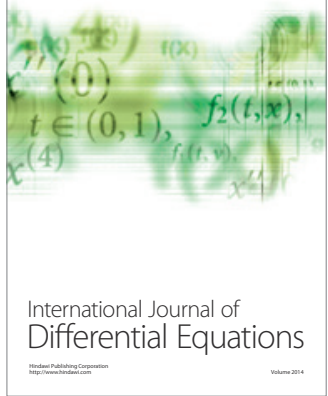
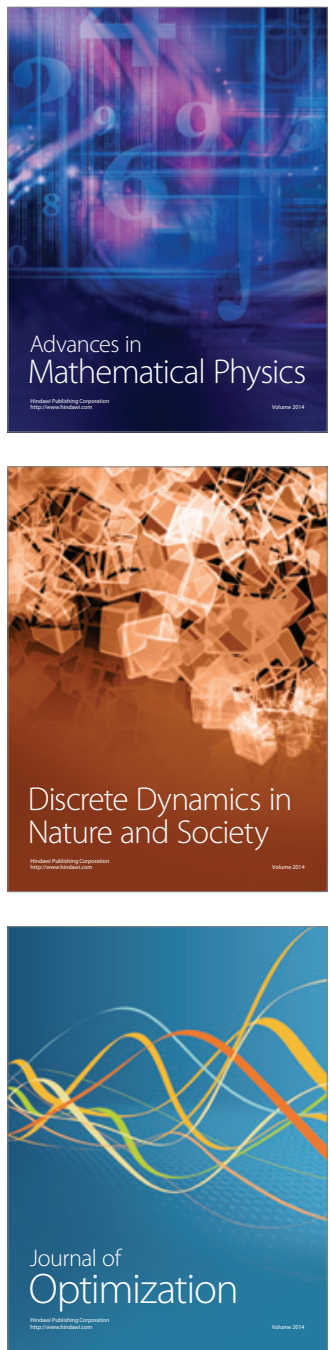